\title{
Efficient Fair TDMA Scheduling In Wireless Multi-Hop Networks
}

\author{
Aggeliki Sgora , Dimitrios J. Vergados, , and Dimitrios D. Vergados \\ University of the Aegean \\ Department of Information and Communication Systems Engineering \\ Karlovassi, Samos, GR-83200,Greece \\ \{asgora; vergados\}@aegean.gr \\ School of Electrical and Computer Engineering \\ National Technical University of Athens \\ GR-15773 Zografou, Athens, Greece \\ djvergad@telecom.ntua.gr
}

\begin{abstract}
Wireless multihop networks have recently been conceived as a networking paradigm. Several algorithms may be found in the literature for scheduling TDMA transmissions for these networks. These algorithms try to determine the optimal scheduling, in order to increase the capacity and reduce the delay for a given network topology. However, to our best knowledge, no TDMA scheduling algorithms have been developed, that take into consideration the traffic requirements of the active flows of the multihop network. At the same time, the fairness of a network is closely related to the scheduling scheme. In this research effort, we propose an intelligent algorithm that can schedule the transmissions in a fair manner, taking into account the communication requirements of the active flows of the network.
\end{abstract}

\section{Introduction}

A wireless multihop network is a network, where communication between twoend nodes is carried out by hopping over multiple short wireless links. In such a network, each node not only sends/receives packets to/from adjacent nodes, but also acts as a router and forwards packets on behalf of other nodes. Wireless multihop networks have many applications, including data monitoring, formation of community and indoor home networks and broadband access network to the Internet. In addition, wireless multihop networks provide wide coverage, as well as, high data rates. The interest in wireless multihop networks has also been increased due to their

Please use the following format when citing this chapter:

Sgora, A., Vergados, D. J., Vergados, D. D., 2007, in IFIP International Federation for Information Processing, Volume 247, Artificial Intelligence and Innovations 2007: From Theory to Applications, eds. Boukis, C., Pnevmatikakis, L., Polymenakos, L., (Boston: Springer), pp. 279-286. 
relatively low deployment costs, since they do not require an infrastructure and complicated network preplanning.

However, despite these advantages, some weaknesses still appear in multi-hop networks. Fairness has been found to be limited in multi-hop networks. Regarding, the spatial-temporal congestion variation, the topology of wireless multi-hop networks and the medium access control protocols that have been designed for single-hop networks, are responsible for severe unfairness in these networks.

In this paper, we focus our study on the medium access control protocols and we propose an algorithm that can schedule the transmissions in a fair manner, taking into consideration the communication requirements of the active flows of the network.

The rest of the paper is organized as follows: Section II discusses the related work, while Section III presents the network model. In Section IV the proposed algorithm is presented and in Section $\mathrm{V}$ an illustrative example is given. Finally, Section VI concludes the paper.

\section{Related Work}

The most popular medium access control scheme for wireless multihop networks is the IEEE 802.11 DCF [1], which uses the CSMA algorithm and has numerous disadvantages like high overhead, increased access delay, high jitter and limited QoS capabilities. Furthermore, 802.11 can not overcome the Exposed Terminal problem. The application of TDMA can overcome all these issues. However, a solution for the NP complete Broadcast Scheduling Problem (BSP) [2] is needed for using TDMA in wireless multihop environment. Several TDMA scheduling algorithms may be found in the literature. [2-7].

More specifically, Ephremides et al [2] in order to solve the problem propose a heuristic algorithm that assumes that each Mobile Station (MS) has knowledge of two hops away connectivity. A priority rule is used to select an eligible MS to transmit in slot $\mathrm{i}$. The selected MS sends a broadcast message to inform the other MSs that it is using slot $i$. The algorithm progresses in a way that allows as many MSs as possible to transmit in each slot. However, it does not ensure fair slot allocations among all MSs and it is not topology-transparent [6]. Also, the authors in [3] propose an approximation algorithm based on Mean Field Annealing (MFA) to solve the scheduling problem and to can achieve maximum channel utilization, as well as, lower delay. More specifically, the authors map the channel utilization to be maximized and the interference-free constraints onto an energy function, and then the MFA procedure is applied to searching for the optimal solutions. Numerical results have shown that the proposed algorithm can find the shortest interference-free frame schedule, while providing the maximum channel utilization. However, the algorithm does not consider the traffic requirements of the active flows of the multihop network. Also, the MFA equations may be also a time consuming process. Salcedo-Sanz et al. [4] propose a mixed neural-genetic algorithm as a solution to the broadcast problem. The proposed algorithm solves the broadcast scheduling problem in two stages: during the first stage finds a feasible frame length able to satisfy 
interference constraints and to guarantee the transmission of every radio station once per frame; the second stage tackles the maximization of the throughput for a given frame length. For the first stage, a discrete Hopfield neural network (HNN) is used and for the second stage, we apply a combination of a HNN and a genetic algorithm (GA). Simulation results show that the proposed algorithm obtains optimum frame lengths and better transmission packings than MFA. However, in this paper only per node fairness is considered. The authors in [5] propose a centralized scheduling algorithm using a modified genetic algorithm (GA), called the genetic-fix algorithm. Particularly, the authors formulate the broadcast scheduling problem as an unconstrained optimization problem. Then, the genetic-fix algorithm is to obtain a conflict-free broadcast assignment where the frame length is close to minimum within a reasonable time. The main advantage of this algorithm at each repetition the search space is reduced. However, the algorithm does not take into consideration the per-flow fairness requirements. Also, in [8] a distributed approach was proposed. In our previous work [7], we proposed an algorithm for overcoming the NP-complete Broadcast Scheduling Problem that appears in TDMA ad-hoc networks. Simulation results showed that the proposed algorithm is superior than the MFA one and equivalent to the mixed neural-genetic algorithm [4] for most of the tested network topologies in terms of delay and throughput, whereas the new algorithm is far superior in terms of fairness. However, the traffic requirements are not considered. In this paper, we propose an algorithm that can schedule the transmissions in a fair manner, taking into consideration the communication requirements of the active flows of a wireless multihop network.

\section{Network Model}

We consider a wireless multihop that consists of a set of $N$ nodes. Every transmission of a node is broadcasted over the wireless channel, and all nodes located close to the transmitting node can receive the transmission, where as far away nodes cannot receive the transmission. Nodes that can transmit with each other are called neighboring nodes. Furthermore, the receiving nodes can only receive one transmission at a time without errors, and nodes cannot transmit and receive packets at the same time.

We assume that multiple access in the wireless channel is achieved by TDMA. All nodes in the wireless multihop network must have at least one transmission opportunity within each TDMA frame. The TDMA frame consists of a number of TDMA slots. The number of slots in each TDMA frame is called the frame length. More than one wireless multihop nodes may transmit in every TDMA slot without collision, if they do not have any common neighbors. The purpose of TDMA scheduling is to determine the slots used by every node for transmitting its packets, in a way that ensures collision avoidance and at the same time minimizes the delay each node experiences, and maximized the total network capacity in a fair manner.

If we represent all nodes in the a wireless multihop network as vertices of a graph with edges between neighboring nodes then there are no collisions in the network, if the distance of all transmitting nodes in the graph is at least two hops. So the 
broadcast scheduling problem is to determine how to schedule every node of the network into the appropriate slot, so that maximum capacity is achieved in the shortest possible frame length. As shown in [2], broadcast scheduling is an NPcomplete problem.

\section{Description of the Proposed Algorithm}

Two stations can use in the same slot if they have no common neighbors. Therefore, we consider the one-hop neighboring table $\mathrm{A}$, where

$$
A_{i, j}=\left\{\begin{array}{cc}
1 & \text { if node } \mathrm{i} \text { and } \mathrm{j} \text { are neighbors, or } \mathrm{i}=\mathrm{j} \\
0 & \text { otherwise }
\end{array} .\right.
$$

We represent the set of nodes transmitting during timeslot $k$ as $S_{k}$. Consider the vector $\mathrm{C}$, which helps to determine if a new node may collide with the slot, where

$$
C_{i}=\sum_{j \in S_{k}} A_{i, j}
$$

If node $\mathrm{k} \notin \mathrm{S}_{\mathrm{k}}$ and there is at least one node $1 \in \mathrm{S}_{\mathrm{k}}$, where $\mathrm{k}$ and 1 have $\mathrm{z}$ as a common neighbour, then $\mathrm{A}_{\mathrm{z}, \mathrm{l}}=\mathrm{A}_{\mathrm{z}, \mathrm{k}}=1$. Therefore, $\mathrm{C}_{\mathrm{z}} \geq 1$ and $\vec{A}_{k} \cdot \vec{C}>0$. On the contrary, if

$$
\vec{A}_{k} \cdot \vec{C}=\mathbf{O}
$$

there is no $l \in S_{k}$ that has a common neighbour with node $k$ and consequently node $\mathrm{k}$ can be added to the slot. This technique is an easy test to determine if a node collides with a node in the TDMA frame, and reduces the complexity of the algorithm.

We also consider the Flow table $\mathrm{F}$, where

$$
F_{i, j}=\left\{\begin{array}{cc}
1 & \text { if node } \mathrm{i} \text { transmits data on behalf of flow } \mathrm{j} \\
0 & \text { otherwise }
\end{array}\right.
$$

In order to produce the desired schedule, all nodes in the network are tested in a specific order. If equation (3) is true for the tested node ( $k$ ), then the node is added to the slot; otherwise it is not added and the next node is tested. When all nodes in the network are tested, then the first time slot is produced. The nodes are re-ordered, and the nodes are re-tested to produce the second timeslot. This procedure is repeated until every node in the network has transmitted as many times as it needs according to the number of flows it participates.

Obviously, the set of nodes in every slot is determined by the order by which the nodes are tested. Since the objectives of the algorithm are fairness we created a weight vector $\vec{W}$, where 


$$
W_{i}=\sum_{j \in T} F_{i, j}
$$

where $T$ is the set of flows in the network. When creating each slot, if $b_{k}$ is the order of node $\mathrm{k}$, then the nodes are ordered as follows:

If $b_{i}<b_{j}$ if $W_{i}>W_{j}$. Nodes that transmit in many slots should be checked first, because nodes that participate in few flows have a grater chance of transmitting in a following slot.

After each slot is created, the values in W are updated and the nodes are reordered. If a slot has been assigned to node $i$ then $W_{i}$ is reduced by 1 , meaning that now it requires one less slot to fulfill its transmission requirements. Then, the next slots are created until all nodes have fulfill their transmission requirements based on the $\mathrm{W}_{\mathrm{i}}$.

\section{An Illustrative Example}

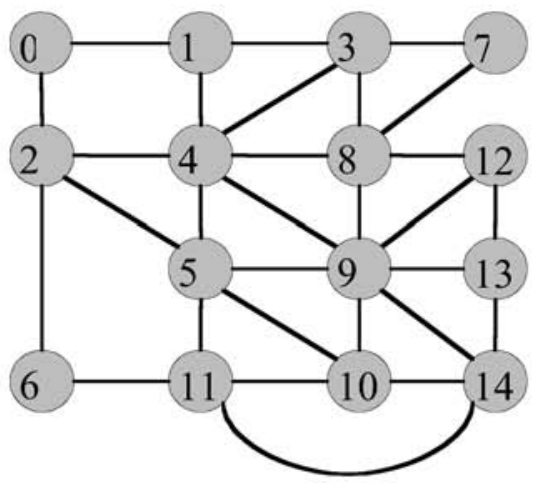

Figure 1. The topology used for the performance evaluation of the algorithm.

In this first approach, we examine the performance of the proposed protocol on a static topology that consists of 15 nodes (Figure 1). In this topology, we randomly selected 6 nodes to be used as sources for the connections, and 6 other nodes to be used as destinations. The sources intend to transmit information to the destinations, and our scheduling scheme will calculate a multihop TDMA schedule, that will be used for organizing the nodes' transmissions, so that no collisions appear, and the frame length is as small as possible. As stated previously, the scheduling scheme will assign the nodes with transmission slots proportionally, depending on the transmission opportunities that each node needs for fulfilling the flows' requirements in a fair manner. 
The produced flows are the following:

- Flow 1: $0-9$

- Flow $2: 2-3$

- Flow $3: 2-12$

- Flow 4: $14-4$

- Flow 5: 7-3

- Flow 6: $6-14$

A shortest path routing protocol was used for obtaining the routes from the sources to the destinations. Note that the shortest path routing algorithm may not always produce the optimal solution, because a different routing could cause a more balanced network, which could result in a shorter frame length. However, this fact is not taken into consideration in this preliminary work, and the shortest paths are used. In future work we will deal with this matter. The routing protocol has produced the following routes:

- Route 1: 0-2-4-9

- Route 2: 2-4-3

- Route 3: 2-4-8-12

- Route 4: 14-9-4

- Route 5: 7-3

- Route 6: 6-11-14

According to the above routes, the corresponding weights $\left(\mathrm{W}_{\mathrm{i}}\right)$ were calculated using equations (4) and (5). As mentioned in the previous section, the scheduling algorithm will assign slots to the nodes, at a number that is proportional to the transmission opportunities required for fulfilling the active flows' needs, as produced by the routing algorithm. The produced weights are shown in Table 1.

Table I. The corresponding weights that are produced by applying the above routes on the network topology

\begin{tabular}{lll}
\hline Node & $\mathrm{W}_{\mathrm{i}}$ \\
\hline 0 & 1 & \\
2 & 3 & \\
4 & 2 & \\
6 & 1 & \\
7 & 1 & \\
8 & 1 & \\
9 & 2 & \\
11 & & 1 \\
14 & 1 & \\
\hline
\end{tabular}

After executing the fair scheduling algorithm, the TDMA schedule was produced (Figure 2). Each row represents a separate slot, and each column represents a node. If the cell that is defined by a node (column) and a slot (row) is white, then the node does not transmit in this slot. Otherwise, if the cell is black, the node will transmit in the slot. As we can see, the produces schedule gives the nodes the appropriate slots. In addition, we see that the nodes may transmit simultaneously, as long this doesn't cause a collision. The produced frame length is 8 . 


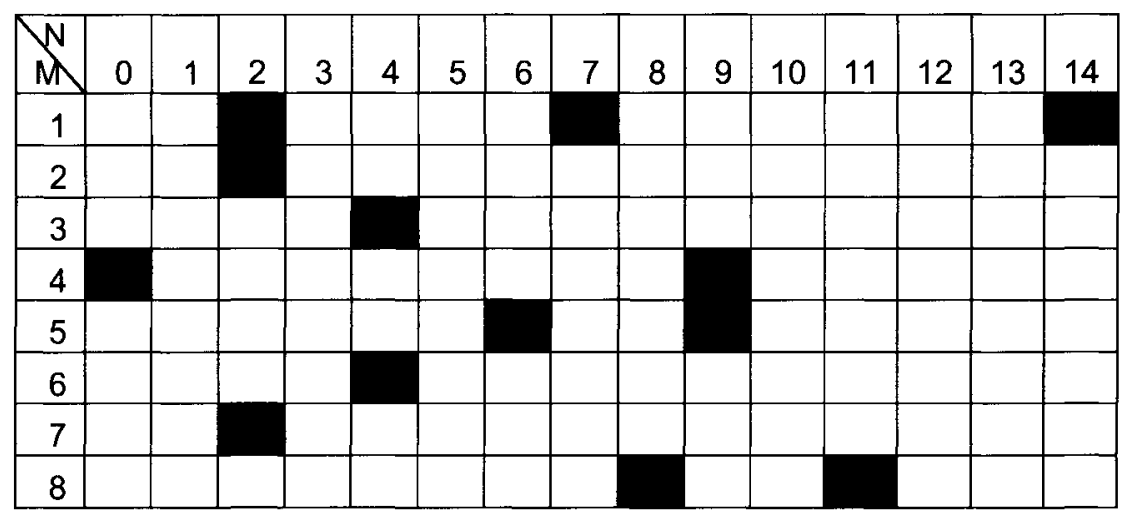

Figure 2. The broadcast schedule for the 14-node-28-edge instance. $\mathrm{N}$ and $\mathrm{M}$ stand for the number of nodes and the number of time slots, respectively. The black square stands for the transmission of node $i$ in slot $j$.

\section{Conclusions}

A wireless multihop network is a network that communication between two end nodes is carried out by hopping over multiple short wireless links. In such network, each node not only sends/receives packets to/from adjacent nodes, but also acts as a router and forwards packets for other nodes. Fairness has been found to be limited in multi-hop networks. The topology of wireless multi-hop networks, in addition to the medium access control protocols that have been designed for single-hop networks, in relation to the spatial-temporal congestion variation are responsible for severe unfairness in these networks. In this paper, we propose an intelligent algorithm that can schedule the transmissions in a fair manner, taking into consideration the communication requirements of the active flows of the network.

\section{References}

1. IEEE std. 802.11, "Wireless LAN Medium Access Control (MAC) and Physical Layer (PHY) Specifications“, 1999.

2. Ephremides and T. V. Truong, "Scheduling broadcast in multihop radio networks," IEEE Transactions on Communications, vol. 38, no. 4, April 1990, pp. 456-460.

3. G. Wang and N. Ansari, "Optimal broadcast scheduling in packet radio networks using mean field annealing," IEEE Journal on Selected Areas in Communications, vol.15, no 2, February 1997, pp.250-260.

4. S. Salcedo-Sanz, C. Busono-Calzon, and A.R. Figueiral-Vidal, "A Mixed Neural-Genetic Algorithm for the Broadcast Scheduling Problem", IEEE Transactions on Wireless Communications, vol. 2, no 2, March 2003, pp. 277-283.

5. Y. Ngo, and Victor O. K. Li, "Centralized Broadcast Scheduling in Packet Radio Networks via Genetic-Fix Algorithms", IEEE Transactions on Communications, vol. 51, no. 9, September 2003, pp. 1439-1441.

6. H. Fattah and C. Leung, "An Overview Of Scheduling Algorithms In Wireless Multimedia Networks”, IEEE Wireless Communications, October 2002, pp. 76-83. 
7. D. J. Vergados, D. D. Vergados and C. Douligeris, "A new approach for TDMA scheduling in ad-hoc networks", In the Proceedings of the I0th IFIP International Conference on Personal Wireless Communications (PWC'05), Colmar, France, 2005.

8. Dimitrios D. Vergados, Dimitrios J. Vergados, Christos Douligeris, Spyridon L. Tombros, "QoS-Aware TDMA for End-to-End Traffic Scheduling in Ad-hoc Networks", IEEE Wireless Communications, Vol. 13, No. 5, 2006, pp. 68-74. 